\title{
La sonorización de las dramatizaciones en el Grado en Educación Infantil
}

\author{
The sound of dramatizations in the Early Childhood Education degree
}

\author{
María Isabel Gómez Núñez \\ migomez@ucam.edu \\ Departamento de Educación \\ Universidad Católica de Murcia \\ Murcia, España \\ ORCID: http://orcid.org/0000-0002-8163-3031 \\ Francisco Javier Marín Marín \\ fjmarin@institutosanfulgencio.es \\ Instituto Teológico San Fulgencio \\ Instituto Superior de Ciencias Religiosas San Fulgencio \\ Murcia, España \\ ORCID: http://orcid.org/0000-0003-4949-9554 \\ José Manuel Azorín Delegido \\ jmazorin@ucam.edu \\ Departamento de Educación \\ Universidad Católica de Murcia \\ Murcia, España \\ ORCID: http://orcid.org/0000-0002-5208-0108
}

doi: 10.7203/LEEME.42.13084

Recibido: 26-09-2018 Aceptado: 30-10-2018. Contacto y correspondencia: José Manuel Azorín Delegido, Departamento de Educación Universidad Católica de Murcia, Campus de los Jerónimos, Guadalupe, Murcia, España

\section{Resumen}

El desarrollo de las nuevas tecnologías y, concretamente, de los medios audiovisuales ha tenido una influencia en la forma de entender la Educación Musical en los centros educativos, así como en la formación inicial de los docentes. Por ello, el propósito principal de este estudio fue evaluar la implementación de dramatizaciones musicales en la asignatura de "Enseñanza y Aprendizaje de la Educación Musical" en el Grado en Educación Infantil de una universidad privada española. La muestra estuvo compuesta por 51 participantes que cursaban el Grado en Educación Infantil en una universidad española. Los resultados mostraron un mayor conocimiento en cuanto al uso de las nuevas tecnologías y de los medios audiovisuales a través del musical mudo. Igualmente, el alumnado valoró positivamente la inclusión de este tipo de ejercicios prácticos en la asignatura. Por tanto, la aplicación de actividades prácticas favorece el desarrollo de una formación inicial de los futuros maestros de Educación Infantil más coherente y adaptada a la realidad de los actuales centros educativos.

Palabras clave: nuevas tecnologías, Educación Infantil, formación inicial docente, Educación Musical.

\begin{abstract}
New technologies and, specifically, audiovisual media development has influenced the way of understanding Music Education in educational centers, as well as in the preservice teachers training. Thus, the aim of this study was to evaluate the implementation of musical dramatizations in the subject of "Teaching and Learning of Music Education" in Early Childhood Education degree programs at a private Spanish university. The sample consisted of 51 participants enrolled in that degree. The results showed a greater knowledge regarding the use of new technologies and audiovisual media through the silent musical. Moreover, the students valued positively the inclusion of this type of practical exercise in the subject. Therefore, the application of practical activities favors the development of a more coherent initial training of the future teachers of Early Childhood Education, adapted to the reality of the current educative centers.
\end{abstract}

Key words: new technologies, Early Childhood Education, teacher initial training, Music Education. 


\section{Introducción}

El desarrollo de Internet y de las nuevas tecnologías de la información y la comunicación (TIC) en los últimos años exigen cambios en el proceso de enseñanza-aprendizaje vinculado a la educación musical que se imparte en las distintas etapas educativas. Según datos del Instituto Nacional de Estadística (INE) (2014), aproximadamente el 93.8\% de los niños con edades comprendidas entre los 10 y los 15 años usan el ordenador habitualmente y, en torno al $92 \%$, emplean también Internet. A estos datos se añade que, tal y como establecen Cánovas, García de Pablo, Oliaga y Aboy (2014), los niños de 2 a 3 años de edad ya tienen acceso periódico a los dispositivos móviles de sus progenitores. Del mismo modo, el incremento de los entornos virtuales de aprendizaje (da Silveira-Borne, 2016) y la importancia de la competencia digital en la sociedad actual abogan por una transformación en la formación inicial docente, si pretendemos que los nuevos maestros de Educación Musical o del resto de especialidades se adapten y respondan a las necesidades de su alumnado. Entre las múltiples ventajas del empleo de las TIC en los distintos periodos educativos destacan el enriquecimiento de los entornos de aprendizaje a través de la participación activa tanto del docente como del alumno (Nart, 2016), así como el incremento de la motivación, la ampliación de la formación auditiva o su complementariedad en las actividades de carácter práctico (Colás-Bravo y Hernández-Portero, 2017).

Estudios como el de Tobias (2013) sugieren que las clases de música deben incluir ejercicios con programas digitales y audiovisuales en los que el alumnado ponga en práctica sus habilidades tecnológicas y musicales. El surgimiento de Youtube y las nuevas tecnologías vinculadas a la creación de vídeos y grabaciones expanden las posibilidades de la educación musical en los estudios superiores. En este sentido, Carrillo-Aguilera y Vilar-Monmany (2014), a través del análisis de documentos y entrevistas al profesorado de música, detectaron tres tipos de competencias que estos debían poseer: competencias profesionales asociadas al desarrollo profesional y a la actuación del docente, competencias musicales relacionadas con la escucha, la interpretación y la creación musical y competencias pedagógicas asociadas a la planificación, aplicación y adaptación de las situaciones de enseñanza-aprendizaje. En todos estos elementos, las TIC emergen como un conjunto de recursos que permiten el desarrollo de la creatividad y de la conciencia de trabajo en equipo-cooperativo, no solo para el docente, sino también para el grupo-clase sobre el que se aplica (Marín-Marín, Gómez-Núñez y Gallego-Moya, 2018).

En el contexto escolar, una de las aplicaciones de los recursos digitales en educación musical es su empleo para la sonorización o musicalización de obras artísticas. Así, estudios como el llevado a cabo por Jara y Martínez (2016) muestran la importancia de incluir la musicalización de obras literarias en el aula ya que favorecen la participación activa del alumnado, la mejora en la lectura comprensiva y expresiva, el desarrollo de la creatividad, así como el incremento de la motivación y de la responsabilidad de llevar a cabo una actividad cooperativa. Además, la sonorización también se emplea para potenciar y aumentar la calidad expresiva de obras visuales como la pintura, permitiendo la creación de una nueva dimensión comunicativa (Bernal-Molina y Aldaz-Casanova, 2017). 
Dada la importancia de las sonorizaciones en la etapa de Educación Primaria, es necesario extrapolar estos procedimientos a la formación inicial y permanente de los futuros docentes de Educación Musical. En este sentido, Galera-Núñez y Mendoza-Ponce (2011) evaluaron el diseño y desarrollo de un proyecto creativo-musical focalizado en la creación de un cuento musical orientado a la etapa de Educación Infantil. La muestra estuvo formada por estudiantes españoles del tercer curso de la titulación Maestro especialista en Educación Musical. Los resultados mostraron que los discentes mejoraron sus habilidades en el manejo de nuevas tecnologías vinculadas con el manejo de sonidos y edición de partituras con un objetivo didáctico. Además, se favoreció el desarrollo de la capacidad creativa y de trabajo en equipo de forma cooperativa de los futuros docentes.

Siguiendo esta línea, Berbel-Gómez y Capellà-Simó (2014) analizaron la puesta en práctica de un proyecto interdisciplinar llevado a cabo con estudiantes universitarios españoles del Grado en Educación Infantil y Primaria. A través de esta propuesta, se vinculaban las asignaturas de Literatura, Música, Artes Plásticas y Nuevas Tecnologías con el fin de construir un relato, el cual sería ilustrado, musicalizado (se compusieron melodías y se incluyeron sonidos ambientales) pasando, finalmente, por un proceso de creación audiovisual en el que se desarrollaría el guion, la digitalización de las ilustraciones, la grabación y el montaje. Los resultados fueron satisfactorios, ya que el estudiantado incrementó su motivación y su capacidad para trabajar en equipo de manera cooperativa.

En otro estudio, Rodríguez-Lorenzo (2014) presenta una propuesta de innovación interdisciplinar llevada a cabo con estudiantes universitarios españoles mediante la que se pretendía, como objetivo principal, la musicalización del anuncio de la campaña "Experimento comparte" (2011) de la organización humanitaria internacional "Acción contra el hambre". Este anuncio trataba de concienciar a la población de la posibilidad de vivir en un mundo sin hambre, evitando la desnutrición infantil. Para la sonorización (musicalización) del anuncio, se emplearon instrumentos y recursos tecnológicos que permitían la edición, interpretación y creación musical. Los resultados mostraron que la utilización del ordenador para crear, editar e incluir la banda sonora del anuncio obligaba a los integrantes de los grupos de trabajo a cooperar y consensuar las decisiones para lograr avanzar en el ejercicio práctico. Igualmente, se observaron mejoras en la creación musical, el incremento en la motivación del alumnado y su participación activa en la elaboración de su anuncio musical.

Por su parte, Cayari (2015) examinó cómo estudiantes universitarios estadounidenses de Educación Musical, creaban vídeos musicales como parte de un proyecto en el que mezclaban música y tecnología. La práctica consistía en sonorizar la letra de canciones creando un vídeo musical similar a los que podemos visualizar en plataformas digitales como Youtube. Los resultados mostraron que los discentes reaccionaron positivamente ante esta práctica, explorando múltiples posibilidades, como los arreglos musicales, versiones, instrumentaciones, etc. El sentimiento de satisfacción y de compañerismo estuvieron presentes en toda la actividad.

A pesar de los resultados satisfactorios de los trabajos mencionados, lo cierto es que la formación inicial en TIC y, concretamente, en medios audiovisuales, continúa siendo escasa en 
nuestras universidades. Así, López-García (2017) observó que el profesorado especialista en Educación Musical, incluido en la muestra de su estudio, afirmó conocer y utilizar las teorías más tradicionales sobre educación musical, obviando recursos como las TIC y/o los medios audiovisuales. Esto se debía no tanto a la falta de recursos en las aulas, sino más bien al déficit en la formación de los docentes, ya fuese por la desmotivación de los mismos hacia estos contenidos o por la baja calidad de los programas de formación en competencias digitales. Estos resultados no solo se dan en las asignaturas vinculadas a la educación musical, sino en la formación general de los futuros maestros de Educación Infantil y Primaria (Roblizo-Colmenero, Sánchez-Pérez y Cózar-Gutiérrez, 2015; Azorín-Delegido y González-Lisorge, 2017), siendo la competencia digital una de las más demandadas por este colectivo (García-Ruiz y Castro-Zubizarreta, 2012).

La importancia de los recursos audiovisuales y tecnológicos, las múltiples posibilidades que ofrecen en cualquier momento y espacio (Gómez-Núñez, Marín-Marín y Cano-Muñoz, 2018), los resultados satisfactorios de investigaciones previas, así como la falta de formación de los docentes de Educación Musical en lo relacionado a herramientas y recursos digitales, justifican la importancia de esta propuesta innovadora y de investigación. Así, el propósito principal fue evaluar la implementación de dramatizaciones musicales en la asignatura de "Enseñanza y Aprendizaje de la Educación Musical" en el Grado en Educación Infantil de una universidad privada española. Específicamente, se esperaba que:

a) El alumnado diseñase e implementase dramatizaciones sonorizadas a través de distintos recursos digitales y musicales.

b) El alumnado valorase positivamente la implementación de esta actividad para la mejora de la calidad de su formación inicial universitaria.

\section{Método}

Este estudio consiste en el diseño y puesta en práctica de una propuesta didáctica en la asignatura de "Enseñanza y Aprendizaje de la Educación Musical" en el Grado en Educación Infantil. Es decir, se trata de un trabajo de aplicación didáctica fundamentada.

\subsection{Muestra}

La selección de la muestra se llevó a cabo a través de un muestreo no probabilístico a propósito o intencional, seleccionando a todo el alumnado que cursaba la asignatura de "Enseñanza y Aprendizaje de la Educación Musical" en tercer curso del Grado en Educación Infantil de una universidad privada española. Así, la muestra estuvo formada por 51 participantes (100\% chicas) con edades comprendidas entre los 20 y los 28 años que cursaban los estudios mencionados durante el curso académico 2017/2018. 


\subsection{Instrumento}

Se elaboró un cuestionario para valorar la calidad de la asignatura y de las distintas partes que la componen. Esta prueba estaba constituida por trece ítems agrupados en dos factores:

1. Valoración de la Titulación (6 ítems; "Valora de forma global los recursos aportados desde la titulación para el desarrollo del plan de estudios").

2. Valoración de la Asignatura (7 ítems; "El profesor fomenta el autoaprendizaje, me guía en la planificación de tareas y orienta correctamente en el desarrollo de las mismas").

Los factores agrupaban ítems referidos a la planificación, metodología, recursos y visión general de la titulación o de la asignatura dependiendo del factor al que hagamos referencia. Estos ítems eran puntuados a través de una escala Likert de cinco puntos $(1=$ Totalmente en desacuerdo; 5 = Totalmente de acuerdo). Las escalas o factores mencionados mostraron índices de consistencia interna (Alpha de Cronbach) en este estudio de .86 (Valoración de la Titulación) y .89 (Valoración de la Asignatura).

La escala de estimación para valorar el rendimiento académico de los discentes en la ejecución del ejercicio práctico propuesto contenía diez ítems (Ejemplo: "La banda sonora del musical está compuesta, al menos, por dos canciones, con una duración aproximada de diez minutos, siendo adecuadas para lo que se desea representar y expresar"), asignando un valor para cada uno de 0 a 1 . La prueba fue construida a través del consenso entre el profesorado de la materia encargado de evaluar la puesta en práctica de la actividad. Esta prueba, completada por el docente, permitió la obtención de una puntuación total, según la ejecución de cada grupo e individuo dentro del musical.

\subsection{Procedimiento}

El profesorado de la asignatura "Enseñanza y Aprendizaje de la Educación Musical" evaluó el musical empleando la escala de estimación durante la ejecución del mismo. Posteriormente, tras esta representación, las alumnas completaron el cuestionario para valorar la planificación, metodología, recursos y visión general de la asignatura en sí misma y de la titulación. De este modo, una vez establecidos los propósitos e hipótesis de trabajo, se llevó a cabo una reunión con las participantes, con el fin de explicarles los motivos de la investigación y solicitar su consentimiento informado por escrito.

El instrumento fue administrado de manera voluntaria y colectiva en el aula a todas las discentes incluidas en la muestra. La administración de la prueba (cuestionario de valoración de la asignatura completado por las alumnas) se llevó a cabo en una sesión de 30 minutos. Los investigadores estuvieron presentes durante la administración de la prueba para aclarar posibles dudas de manera individual, asegurándose que los datos fuesen debidamente anotados y agradeciendo la colaboración de todas las participantes. 


\subsection{Materiales y desarrollo de la actividad}

Se preparó un dosier con las instrucciones precisas para la realización de la práctica. En este documento se contemplan los objetivos generales, las competencias generales y específicas, así como los resultados de aprendizaje de la asignatura. Del mismo modo, se incluyen los aspectos generales, recursos, formato e instrucciones del ejercicio práctico que las alumnas debían desarrollar (el musical mudo en Educación Infantil).

La práctica de la que parte este estudio consistió en la planificación y representación real de un musical mudo sobre un cuento infantil. Las instrucciones para su desarrollo se exponen a continuación:

1. División de la clase en grupos de 6-10 personas.

2. Selección de un cuento infantil que fue representado en forma de musical mudo en el aula.

3. Distribución de los personajes del cuento entre los componentes del grupo. Si existían suficientes personajes para todos los integrantes, se ofreció la posibilidad de añadir más personajes (inventados) o de establecer un elenco de personajes secundarios-coro. Los personajes secundarios-coro aluden a un conjunto de actores que no adoptan un papel protagonista en la historia pero que intervienen en su desarrollo, ayudando a los personajes principales y ofreciendo una mayor riqueza a lo representado.

4. Concreción del guión de nuestra historia-cuento musical que permitía guiar la trama y lo que se pretendía representar (podían suprimirse o ampliarse partes del cuento seleccionado).

5. Selección de dos canciones (podían ser actuales). Una de las canciones debía estar en modo Mayor, mientras que la otra canción debía presentarse en modo menor. Se modificó la letra de las canciones seleccionadas para ajustarlas al cuento-historia que se relataba a través del musical. Esta parte era muy importante, ya que constituía una de las pocas ocasiones en las que los personajes podían expresarse a través de la palabra. Las canciones debían contar con la base de sonido de fondo.

6. Las canciones fueron cantadas a lo largo de la representación-musical cuando los componentes del equipo estimaron conveniente, en función del desarrollo de la historia.

7. Planificación y ejecución de dos danzas. Las danzas se adaptaron al nivel de competencia curricular de la etapa de Educación Infantil, evitando movimientos y pasos demasiado técnicos, más propios de la danza practicada por los adultos. La música seleccionada para cada una de las danzas debía tener las siguientes características (a elegir entre la opción a y b): a) una pieza musical-instrumental (sin letra); una pieza musical-cantada; b) dos piezas musicales-instrumentales (sin letra).

8. Cada una de las danzas fue ejecutada a lo largo de la representación-musical cuando los componentes del equipo estimaron conveniente, en función del desarrollo de la historia.

9. Selección de un mínimo de tres instrumentos musicales que fueron tocados a lo largo del musical. Estos instrumentos podían ser de elaboración propia o de los existentes en el

@MARÍA ISABEL GÓMEZ NÚÑEZ, FRANCISCO JAVIER MARIN MARÍN Y JOSÉ MANUEL AZORIN DELEGIDO. THE CONTENT OF THIS ARTICLE IS THE SOLE RESPONSIBILITY OF THE AUTHORS. THE REVISTA ELECTRÓNICA DE LEEME AND UNIVERSITAT DE VALĖNCIA ARE NOT LIABLE FOR ANY LEGAL ACTIONS THAT MAY ARISE INVOLVING THE ARTICLE'S CONTENT. REVISTA ELECTRÓNICA DE LEEME -LISTA ELECTRÓNICA EUROPEA DE MÚSICA EN LA EDUCACIÓN-HTTP://OJS.UV.ES/INDEX.PHP/LEEME/INDEX. ISSN: 1575-9563 EDITORES: UNIVERSIDAD DE VALENCIA Y JESÚS TEJADA GIMÉNEZ. VISIBILIDAD DE ESTA REVISTA: SCOPUS, EMERGING SOURCES CITATION INDEX (CLARIVATE), EBSCO, CINDOC (CSIC), CITEFACTOR, COPAC, JALNA DICE (CSIC), DOAJ, E-REVISTAS (CSIC), EBSCO PREMIER, ERIH+, GALE CENGAGE LEARNING, IN-RECS, IRESIE, LATINDEX, MIAR, OCLC WORLDCAT, REFI, REDIB, RILM CORE
JOURNALS, SUDOC, ULRICHS, ESTA REVISTA ESTA PUBLICADA CON EL APOYO INSTTTUCIONAL DE REDIRIS-CONSEJO SUPERIOR DE INVESTIGACIONES CIENTIFICAS Y ES DE ACCESO LIBRE. CREATIVE COMMONS LICENSE $4.0 \mathrm{BY}$ 
aula de música. Los instrumentos funcionaban como acompañamiento en las canciones interpretadas o simulando sonidos ambientales, sonidos propios del cuento, etc.

10. Selección de un mínimo de seis efectos audiovisuales incluidos a lo largo de la representación.

11. Banda Sonora: utilización de una banda sonora de fondo a lo largo de la representación, la cual debía estar compuesta por un mínimo de dos canciones/obras (una de ellas asociada a emociones positivas y otra asociada a emociones negativas). El apoyo musical era imprescindible a lo largo del trabajo, por lo que debía estar presente durante al menos diez minutos (sin contar el tiempo dedicado a las danzas y a las canciones interpretadas). La banda sonora no incluía los efectos o sonidos ambientales, sino únicamente las piezas u obras musicales seleccionadas.

12. Durante toda la exposición quedaron representadas distintas emociones (miedo, ira, alegría, tristeza, sorpresa, etc.) utilizando, para ello, tanto el trabajo corporal como la música.

13. Reflejar contrastes de intensidad (fuerte-débil), altura (agudo-grave) y velocidad (rápidolento).

14. No se permitía el diálogo dentro del musical. Solo se permitía, al comienzo del mismo, una breve narración que sirviera como introducción de la representación.

15. Se permitieron las onomatopeyas o sonidos silábicos.

16. Durante gran parte de la representación debían estar "en escena", al menos, la mitad de los miembros del grupo. Evitar "protagonismos excesivos" de alguno/s de los miembros del grupo.

17. Solo se podía utilizar atrezo, maquillaje y vestimentas elaboradas con material del aula (cartulinas, bolsas de basura, cosas que tengamos en casa, etc.).

18. El decorado se elaboró con materiales propios del aula: cartulinas, cartones, goma eva, papel continuo, etc. No se podían comprar materiales adicionales ni traer medios tecnológicos adicionales que excedieran los propósitos de esta práctica y los recursos que encontramos en un aula en Educación Infantil.

19. La duración del musical fue de 25 minutos (con 5 minutos de margen).

20. Elaboración de un tríptico como guión para las personas que viesen cada musical. La elaboración del mismo dependía de la imaginación del grupo y de lo que quisieran expresar en él. El texto incluido en el tríptico fue revisado por los docentes de la asignatura, con el fin de que corregir errores y verificar que la información ofrecida resultara relevante y coherente.

Se partió de una base teórica previa que permitiera a las alumnas contar con los conocimientos adecuados para planificar y desarrollar por grupos su musical mudo. Dicha planificación tuvo una duración de 20 sesiones de sesenta minutos a lo largo del segundo cuatrimestre (Febrero-Junio) del curso académico 2017/2018.

La primera sesión se dedicó a la elección del cuento y al comienzo de la adaptación del mismo para convertirlo en un musical. Para ello, se realizó una selección previa de cuentos, atendiendo a los que las alumnas conocían de asignaturas vinculadas con la literatura infantil en

@MARÍA ISABEL GÓMEZ NúŃEZ, FRANCISCO JAVIER MARIN MARÍN Y JOSÉ MANUEL AZORIN DELEGIDO. THE CONTENT OF THIS ARTICLE IS THE SOLE RESPONSIBILITY OF THE AUTHORS. THE REVISTA ELECTRÓNICA DE LEEME AND UNIVERSITAT DE VALÈNCIA ARE NOT LIABLE FOR ANY LEGAL ACTIONS THAT MAY ARISE INVOLVING THE ARTICLE'S CONTENT. REVISTA ELECTRÓNICA DELEEME-LISTA ELECTRONICA EUROPEA DE MÚSICA ENLA EDUCACION-HTTP://OJSUVVES/INDEX.PHP/LEEME/INDEX, ISSN: 1575-9563 EDITORES: UNIVERSIDAD DE VALENCIA Y JESÚS TEJADA GIMÉNEZ. VISIBILIDAD DE ESTA REVISTA: SCOPUS, EMERGING SOURCES CITATION INDEX (CLARIVATE), EBSCO, CINDOC (CSIC), CITEFACTOR, COPAC, DIALNET, DICE (CSIC), DOAJ, E-REVISTAS (CSIC), EBSCO PREMIER, ERIH+, GALE CENGAGE LEARNING, IN-RECS, IRESIE, LATINDEX, MIAR, OCLC WORLDCAT, RESH, REDIB, RILM CORE JOURNALS, SUDOC, ULRICHS, ESTA REVISTA ESTÁ PUBLICADA CON EL APOYO INSTITUCIONAL DE REDIRIS-CONSEJO SUPERIOR DE INVESTIGACIONES CIENTIFICAS Y ES DE ACCESO LIBRE. CREATIVE COMMONS LICENSE 4.0 BY 
el grado cursado. En la segunda sesión, los distintos grupos de clase continuaron con la adaptación del cuento y la distribución de personajes.

La tercera, cuarta y quinta sesiones se destinaron a la selección de canciones (modo mayor y modo menor) y al cambio de la letra de las mismas, con el fin de adaptarlas a la historia contada por cada uno de los grupos de trabajo. A la hora de cambiar la letra de las canciones por otra adaptada a la historia que cada grupo contaba, se debía analizar la canción original para identificar la acentuación. Posteriormente, se procedió a la creación de un texto acorde a la acentuación de los compases que componían las distintas melodías y al cuento escogido, haciendo especial hincapié en la coincidencia entre los acentos de texto y música.

La sexta, séptima y octava sesiones se enfocaron a la planificación de las danzas y de la expresión corporal que debía predominar a lo largo de la representación. Para ello, en primer lugar, se seleccionaron las canciones u obras que se dramatizarían. En segundo lugar, se analizaron los tiempos y la forma musical de cada una de ellas, con el fin de adecuar los pasos a su estructura. En este sentido, se dieron indicaciones al alumnado sobre la importancia de incluir pasos sencillos que se repitieran y estuvieran adaptados a las capacidades de los niños de la etapa de Educación Infantil.

La novena sesión se destinó a la práctica con los instrumentos musicales que se introducirían en cada historia. Cada grupo seleccionó un mínimo de tres instrumentos musicales de percusión cuyo timbre resultara adecuado al momento de la historia en el que sería introducido. Además, se facilitaron las instrucciones pertinentes en cuanto a la técnica instrumental necesaria.

La décima y undécimas sesiones se centraron en la planificación de los efectos audiovisuales. Dentro de estas clases se ofrecieron bancos de sonidos online donde las alumnas investigaron los más apropiados para su historia.

La duodécima y décimo-tercera sesiones se destinaron a la selección de la banda sonora y su adaptación al musical, por lo que los discentes tuvieron que seleccionar fragmentos, unirlos y formar la que sería la música de su representación. Así, se facilitaron enlaces a distintas obras que podían ser incluidas, indicando que estas podrían ser ampliadas en función de las necesidades del cuento. En este sentido, se incidió en la necesidad de que la música se vinculase con el momento representado a través de cambios de dinámica, de modalidad, de agógica, etc.

Las sesiones décimo-cuarta, décimo-quinta y décimo-sexta se dedicaron a la elaboración del decorado y de las vestimentas que cada grupo llevaría en su representación. Y, en las siguientes tres sesiones, se fijaron ensayos generales en distintas aulas, con el fin de que todos los grupos tuvieran las mismas oportunidades para practicar su ejecución final. Por último, se realizó la representación de cada uno de los musicales, permitiendo al alumnado que invitaran a sus familiares, amigos o docentes.

@MARÍA ISABEL GÓMEZ NÚÑEZ, FRANCISCO JAVIER MARÍN MARÍN Y JOSÉ MANUEL AZORIN DELEGIDO. THE CONTENT OF THIS ARTICLE IS THE SOLE RESPONSIBILITY OF THE AUTHORS. THE REVISTA ELECTRÓNICA DE LEEME AND UNIVERSITAT DE VALĖNCIA ARE NOT LIABLE FOR ANY LEGAL ACTIONS THAT MAY ARISE INVOLVING THE ARTICLE'S CONTENT. REVISTA ELECTRÓNICA DE LEEME -LISTA ELECTRÓNICA EUROPEA DE MÚSICA EN LA EDUCACIÓN-HTTP:// OJS.UV.ES/INDEX.PHP/LEEME/INDEX. ISSN: 1575-9563 EDITORES: UNIVERSIDAD DE VALENCIA Y JESÚS TEJADA GIMÉNEZ. VISIBILIDAD DE ESTA REVISTA: SCOPUS, EMERGING SOURCES CITATION INDEX (CLARIVATE), EBSCO, CINDOC (CSIC), CITEFACTOR, COPAC,
DIALNET, DICE (CSIC), DOAJ, E-REVISTAS (CSIC), EBSCO PREMIER, ERIH+, GALE CENGAGE LEARNING, IN-RECS, IRESIE, LATINDEX, MIAR, OCLC WORLDCAT, RESH, REDIB, RILM CORE JOURNALS, SUDOC, ULRICHS, ESTA REVISTA ESTÁ PUBLICADA CON EL APOYO INSTITUCIONAL DE REDIRIS-CONSEJO SUPERIOR DE INVESTIGACIONES CIENTIFICAS Y ES DE ACCESO LIBRE. CREATIVE COMMONS LICENSE 4.O BY 


\section{Resultados}

La Figura 1 muestra los resultados obtenidos en cuanto al rendimiento total de las estudiantes en la ejecución del ejercicio práctico:

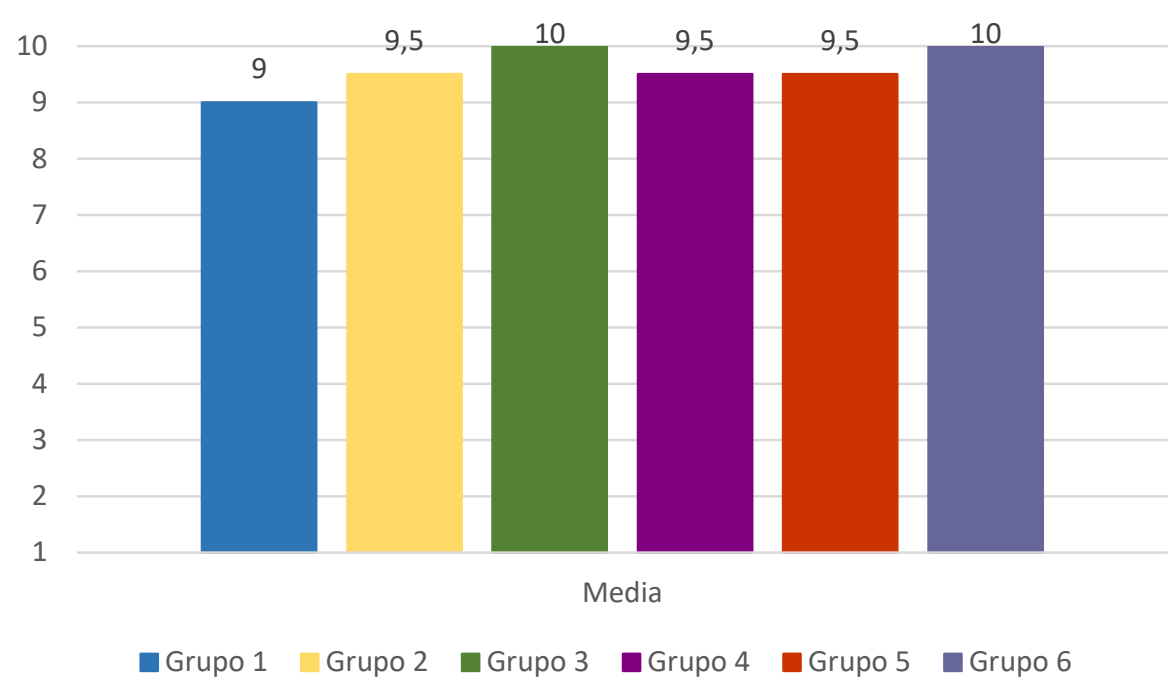

Figura 1. Nota media en el ejercicio práctico planteado (puntuación media sobre 10)

La nota media de los distintos grupos osciló entre 9 puntos (Grupo 1) y 10 puntos (Grupos 3 y 6), teniendo en cuenta la calificación total obtenida a partir de la suma de puntos conseguidos en cada uno de los indicadores propuestos en la escala de estimación que permitía la evaluación de la representación final del musical. Los indicadores en los que los distintos grupos adquirieron mayor puntuación (un punto máximo) fueron los referidos a la expresión de emociones (mostraron una gran variedad de emociones positivas y negativas vinculadas a la historia representada), contrastes musicales (intensidad, altura y velocidad), la adaptación de las canciones (la letra se adecuaba tanto al cuento, como a la acentuación musical de la melodía), la ejecución de las danzas (inclusión de pasos sencillos que se repetían acordes al ritmo de las obras elegidas), la selección de la banda sonora del musical (variada, coherente con lo que se representaba y con la duración propuesta en las instrucciones) y la integración de los recursos audiovisuales en la dramatización.

La Figura 2 muestra los resultados vinculados a la valoración de la asignatura por parte del alumnado:

@MARÍA ISABEL GÓMEZ NÚÑEZ, FRANCISCO JAVIER MARÍN MARÍN Y JOSÉ MANUEL AZORIN DELEGIDO. THE CONTENT OF THIS ARTICLE IS THE SOLE RESPONSIBILITY OF THE AUTHORS. THE REVISTA ELECTRÓNICA DE LEEME AND UNIVERSITAT DE VALĖNCIA ARE NOT LIABLE FOR ANY LEGAL ACTIONS THAT MAY ARISE INVOLVING THE ARTICLE'S CONTENT. REVISTA ELECTRÓNICA DE LEEME - LISTA ELECTRÓNICA EUROPEA DE MÚSICA EN LA EDUCACIÓN-HTTP:/ /OJS.UV.ES/INDEX.PHP/LEEME/INDEX. ISSN: 1575-9563 EDITORES: UNIVERSIDAD DE VALENCIA Y JESÚS TEJADA GIMÉNEZ. VISIBILIDAD DE ESTA REVISTA: SCOPUS, EMERGING SOURCES CITATION INDEX (CLARIVATE), EBSCO, CINDOC (CSIC), CITEFACTOR, COPAC, JOURNALS, SUDOC, ULRICHS, ESTA REVISTA ESTA PUBLICADA CON EL APOYO INSTITUCIONAL DE REDIRIS-CONSEJO SUPERIOR DE INVESTIGACIONES CIENTIFICAS Y ES DE ACCESO LIBRE. CREATIVE COMMONS LICENSE $4.0 \mathrm{BY}$ 


\section{Media}

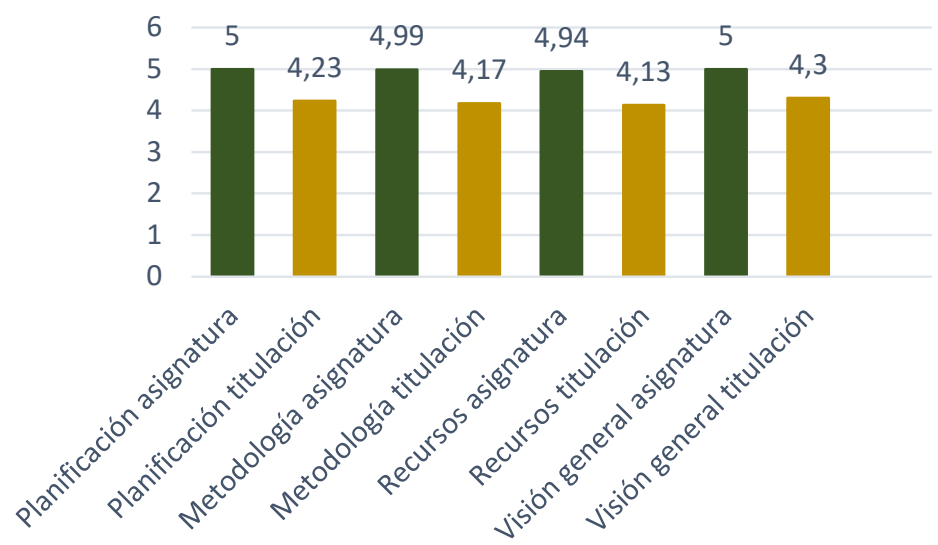

Figura 2. Valoración de la asignatura y de la titulación (puntuación media sobre 5)

La puntuación media $(M)$ respecto a la valoración que el alumnado hizo de la asignatura osciló entre 4.94 (recursos de la asignatura) y 5 (planificación y visión general de la materia). Estas puntuaciones medias $(M)$ fueron superiores a las otorgadas para la titulación, las cuales oscilaron entre 4.13 (recursos de la titulación) y 4.3 (visión general de la titulación). La puntuación media $(M)$ de la asignatura fue de $4.98(D T=1.22)$, mientras que la puntuación media $(M)$ de la titulación fue $4.21(D T=1.23)$.

\section{Discusión}

El objetivo general de este estudio fue evaluar la implementación de musicales mudos con medios audiovisuales en la asignatura de Enseñanza y Aprendizaje de la Educación Musical en el Grado en Educación infantil.

Según la primera hipótesis planteada, se esperaba que las alumnas del Grado en Educación Infantil diseñasen e implementasen dramatizaciones sonorizadas a través de distintos recursos digitales y musicales. Los resultados mostraron que esta hipótesis se confirmaba en todos los grupos de trabajo formados en el aula, ya que los participantes obtuvieron medias superiores a cinco puntos (puntuación límite para superar el ejercicio práctico). Esto indicaba que los musicales cumplieron con los requisitos establecidos en el dosier o documento con las instrucciones pertinentes, lo que daba muestras del adecuado diseño e implementación de la dramatizaciones sonorizadas mediante diversas herramientas digitales y musicales (Youtube, grabaciones, karaoke, programas informáticos especializados, instrumentos, etc.), así como de la comprensión de los contenidos teóricos de la asignatura, lo cual resultaba coherente con investigaciones previas en las que estudiantes universitarios unían música y tecnología para la sonorización de historias, relatos o letras dentro de sus estudios en Educación Musical (Galera- 
Núñez y Mendoza-Ponce, 2011; Rodríguez-Lorenzo, 2014; Berbel-Gómez y Capellà-Simó, 2014; Cayari, 2015).

Las puntuaciones más altas se obtuvieron en lo referido a la variación de estados emocionales y contrastes musicales, la elaboración y adecuación de música y texto en la adaptación de canciones, la expresión corporal, la elección de los fragmentos musicales utilizados a lo largo de la representación y el empleo de recursos digitales básicos para la sonorización del musical. Estos procedimientos son fundamentales en la etapa de Educación Primaria, tal y como refuerzan estudios basados en la sonorización de obras literarias y pictóricas (Jara y Martínez, 2016; Bernal-Molina y Aldaz-Casanova, 2017). Además, el desarrollo de competencias musicales tan importantes como las vinculadas con la escucha, la interpretación y la creación musical (Carrillo-Aguilera y Vilar-Monmany, 2014) precisan, en la actualidad, del empleo de las TIC como una de sus herramientas básicas (Galera-Núñez y Mendoza-Ponce, 2011).

La segunda hipótesis, por la que se esperaba que las alumnas valorasen positivamente la implementación de la actividad de los musicales para la mejora de la calidad de su formación inicial universitaria, también quedó confirmada. Los hallazgos mostraron puntuaciones cercanas o iguales a cinco (puntuación máxima) en todos los elementos evaluados (planificación, metodología, recursos y visión general de la asignatura). Estas puntuaciones fueron superiores a la media obtenida por la titulación, lo que dio muestras de la importancia que las alumnas concedían al planteamiento práctico de la materia. La necesidad de incluir programas digitales y audiovisuales en las clases de música (Tobias, 2013), se hace patente por los cambios en la educación musical derivados del desarrollo de Internet y de las TIC, por el incremento del uso temprano de Internet, móviles u ordenadores en la infancia (Cánovas et al., 2014; INE, 2014), así como por el auge de los entornos virtuales de aprendizaje en la formación inicial de los docentes (Da Silveira-Borne, 2016). Igualmente, los maestros de Educación Infantil y Primaria en general y aquellos que imparten la asignatura de Educación Musical en particular, reclaman una mayor formación inicial en estos nuevos recursos digitales (Roblizo et al., 2015; Azorín-Delegido y González-Lisorge, 2017; López-García, 2017) ya que favorecen la participación activa del docente y del alumno (Nart, 2016), el aumento de su motivación y la mejora de su formación auditiva (Colás-Bravo y Hernández-Portero, 2017).

A pesar de la importancia de los resultados obtenidos, este estudio no está exento de una serie de limitaciones que deberán ser tenidas en cuenta en futuras investigaciones en este ámbito. Así, es necesario señalar que la muestra, seleccionada a propósito, pertenece a una única universidad española, por lo que sería aconsejable extrapolar esta práctica a otras universidades y grados en los que se imparta la materia de Educación Musical. Igualmente, no se han tenido en cuenta otras competencias o variables psicoeducativas distintas a las mencionadas y que también podrían influir en los resultados de esta investigación. Por último, la realización de un estudio transversal no nos permitió evaluar si estos aprendizajes influyen en la práctica real de los maestros en los centros de Educación Infantil y Primaria. En este sentido, sería interesante la realización de estudios longitudinales que posibilitarán analizar si los conocimientos adquiridos

@MARIA ISABEL GÓMEZ NúÑEZ, FRANCISCO JAVIER MARÍN MARÍN Y JOSÉ MANUEL AZORIN DELEGIDO. THE CONTENT OF THIS ARTICLE IS THE SOLE RESPONSIBILITY OF THE AUTHORS, THE REVISTA ELECTRÓNICA DE LEEME AND UNIVERSITAT DE VALĖNCIA ARE NOT LIABLE FOR ANY LEGAL ACTIONS THAT MAY ARISE INVOLVING THE ARTICLE'S CONTENT. REVISTA ELECTRÓNICA DE LEEME -LISTA ELECTRÓNICA EUROPEA DE MÚSICA EN LA EDUCACIÓN-HTTP:// OJS.UV.ES/INDEX.PHP/LEEME/INDEX. ISSN: 1575-9563 EDITORES: UNIVERSIDAD DE VALENCIA Y JESÚS TEJADA GIMÉNEZ. VISIBILIDAD DE ESTA REVISTA: SCOPUS, EMERGING SOURCES CITATION INDEX (CLARIVATE), EBSCO, CINDOC (CSIC), CITEFACTOR, COPAC, JOURNALS, SUDOC, ULRICHS, ESTA REVISTA ESTÁ PUBLICADA CON EL APOYO INSTITUCIONAL DE REDIRIS-CONSEJO SUPERIOR DE INVESTIGACIONES CIENTIFICAS Y ES DE ACCESO LIBRE. CREATIVE COMMONS LICENSE 4.0 BY 
en la formación inicial en cuanto a medios digitales y/o audiovisuales se ponen en práctica en la labor docente posterior.

\section{Conclusiones}

Las TIC y, concretamente, los medios audiovisuales aplicados a la enseñanza musical emergen como uno de los recursos más importantes y adaptados a la sociedad de la información actual (García-Ruiz y Castro-Zubizarreta, 2012).

En primer lugar, es necesario destacar el desarrollo de la creatividad en el alumnado, la cooperación entre los mismos y las múltiples posibilidades que estos medios nos ofrecen, los cuales hacen necesaria su inclusión en la formación inicial del futuro profesorado de Educación Musical (Gómez-Núñez et al., 2018; Marín-Marín et al., 2018).

En segundo lugar y siguiendo esta línea, los resultados de esta investigación, de carácter práctico y descriptivo, ponen de manifiesto la importancia de incluir contenidos específicos vinculados con las TIC en los planes de estudio de los Grados en Educación Infantil y Educación Primaria, no solo porque favorecen el desarrollo de la competencia digital de los maestros, básica para adaptarse a la sociedad actual, sino también porque constituye el primer paso para construir entornos educativos que cubran las necesidades del alumnado.

Por último, la investigación realizada permitió cumplir el objetivo general propuesto, siendo el de evaluar la implementación de dramatizaciones musicales en la asignatura de "Enseñanza y Aprendizaje de la Educación Musical" en el Grado en Educación infantil. Las hipótesis se confirmaron ya que las alumnas diseñaron e implementaron dramatizaciones sonorizadas a través de distintos recursos digitales y musicales, valorando positivamente la calidad de su formación.

La puesta en práctica de este tipo de actividades enfrenta a los futuros docentes de Educación Musical a una de las actividades que podrán desarrollar en la etapa de Educación Primaria: el empleo de la música para sonorizar relatos u otro tipo de obras artísticas (Jara y Martínez, 2016; Bernal-Molina y Aldaz-Casanova, 2017) favoreciendo, de este modo, la unión entre la formación inicial y el desempeño en la práctica real.

\section{Referencias}

Azorín-Delegido, J. M. y González-Lisorge, S. (2017). El diseño de una página web en el proceso de enseñanza-aprendizaje en la Educación Superior. Diferencias entre modalidad presencial y semipresencial. En J. E. Gonzálvez-Vallés (Coord.), Nuevas tendencias en innovación educativa superior (pp. 105-124). Madrid: ACCI. 
Berbel-Gómez, N. y Capellà-Simó, P. (2014). Cuentos musicales ilustrados y con soporte audiovisual: Una experiencia interdisciplinar en los estudios de grado de educación infantil y primaria. DEDiCA. Revista De Educação e Humanidades, 6, 287-294.

Bernal-Molina, A. y Aldaz-Casanova, C. (2017). Prolongación del alcance del significado de una obra plástica (o visual) mediante su sonorización. En E. M. Pérez García (Presidente), III Congreso Internacional de Investigación en Artes Visuales. Valencia: ANIAV. doi:10.4995/ANIAV.2017.4930

Cánovas, G., García de Pablo, A., Oliaga, A. y Aboy, I. (2014). Menores de edad y conectividad móvil en españa: Tablets y smartphones. Centro De Seguridad En Internet Para Los Menores En España: PROTEGELES, Dependiente Del Safer Internet Programa De La Comisión Europea. Recuperado de: http://www.Diainternetsegura.es/

Carrillo-Aguilera, C. C. y Vilar-Monmany, M. V. (2014). El perfil profesional del profesorado de música: Una propuesta de las competencias deseables en ed. primaria y ed. secundaria. Revista Electrónica de LEEME, 33, 1-26. doi:10.7203/LEEME.39.9904

Cayari, C. (2015). Participatory culture and informal music learning through video creation in the curriculum. International Journal of Community Music, 8 (1), 41-57. doi:10.1386/ijcm.8.1.41_1

Colás-Bravo, P. y Hernández-Portero, G. (2017). Itinerarios formativos del profesorado de Música: sus percepciones sobre el valor didáctico de las TIC. Revista Fuentes, 19 (1), 39-56. doi: doi: 10.12795/revistafuentes. 2017.19.1.02

Da Silveira-Borne, L. (2016). Tecnologías en la educación musical a distancia en contextos universitarios brasileños. Cuadernos De Música, Artes Visuales y Artes Escénicas, 11 (1), 131150. doi: 10.11144/Javeriana.mavae11-1.temd

Galera-Núñez, M. M. y Mendoza-Ponce, J. (2011). Tecnología Musical y Creatividad: Una experiencia en la formación de maestros. Revista Electrónica De LEEME, (28), 24-36. doi: 10.7203/LEEME.39.9904

García-Ruiz, R. y Castro-Zubizarreta, A. (2012). La formación permanente del profesorado basada en competencias. Estudio exploratorio de la percepción del profesorado de Educación Infantil y Primaria. Educatio Siglo XXI, 30 (1), 297-322.

Gómez-Núñez, M. I., Marín-Marín, F. J. y Cano-Muñoz, M. A. (2018). Recursos didácticos en lenguaje musical. En M. I. Gómez-Núñez y F. J. Marín-Marín (Coords.), Lenguaje Musical (pp. 129-139). Murcia: Diego Marín.

Instituto Nacional de Estadística (2014). Encuesta sobre equipamiento y uso de Tecnologías de Información y Comunicación en los Hogares. Recuperado de http://www.ine.es/jaxi/menu.do?type=pcaxisypath=/t25/p450/base_2011/a2014/yfile=pcaxis 
Jara, M. I. D. V. Y. y Martínez, M. M. (2016). La dramatización musical del romancero en Educación Primaria para el desarrollo de la competencia social y ciudadana. Educatio Siglo XXI, 34(1), 11-30. doi: 10.6018/j/252511

López-García, N. J. (2015). Necesidades profesionales del profesorado especialista de música de los centros de educación primaria de Castilla-la Mancha. Revista Electrónica De LEEME, (39), 74-102. doi: 10.7203/LEEME.39.9904

Marín-Marín, F. J., Gómez-Núñez, M. I. y Gallego-Moya, J. (2018). Las TIC en el aula de música. En F. J. Marín-Marín y M. I. Gómez-Núñez, Metodología de la Enseñanza Musical (pp. 75-87). Murcia: Diego Marín.

Nart, S. (2016). Music Software in the Technology Integrated Music Education. TOJET: The Turkish Online Journal of Educational Technology, 15 (2), 78-84.

Roblizo-Colmenero, M. J. Sánchez-Pérez, M. C. y Cózar-Gutiérrez, R. (2015). El reto de la competencia digital en los futuros docentes de infantil, primaria y secundaria: Los estudiantes de grado y máster de educación ante las TIC. Prisma Social: Revista de Investigación Social, 15, 254-295.

Rodríguez-Lorenzo, G. A. (2014). La creación musical cooperativa y las II.II.CC.: una propuesta para la Educación Musical Universitaria. En J. F. Durán-Medina y S. Durán-Valero, La era de las TT.II.CC. en la nueva docencia (pp. 435-450). Madrid: McGraw-Hill Interamericana de España.

Tobias, E. S. (2013). Toward convergence: Adapting music education to contemporary society and participatory culture. Music Educators Journal, 99 (4), 29-36. doi: $10.1177 / 0027432113483318$

@MARÍA ISABEL GómEZ NúÑEZ, FRANCISCO JAVIER MARÍN MARÍN Y JOSÉ MANUEL AZORIN DELEGIDO. THE CONTENT OF THIS ARTICLE IS THE SOLE RESPONSIBILITY OF THE AUTHORS. THE REVISTA ELECTRÓNICA DE LEEME AND UNIVERSITAT DE VALĖNCIA ARE NOT LIABLE FOR ANY LEGAL ACTIONS THAT MAY ARISE INVOLVING THE ARTICLE'S CONTENT. REVISTA ELECTRÓNICA DE LEEME -LISTA ELECTRÓNICA EUROPEA DE MÚSICA EN LA EDUCACIÓN-HTTP://OJS.UV.ES/INDEX.PHP/LEEME/INDEX. ISSN: 1575-9563 EDITORES: UNIVERSIDAD DE VALENCIA Y JESÚS TEJADA GIMÉNEZ. VISIBILIDAD DE ESTA REVISTA: SCOPUS, EMERGING SOURCES CITATION INDEX (CLARIVATE), EBSCO, CINDOC (CSIC), CITEFACTOR, COPAC, JOURNALS, SUDOC, ULRICHS, ESTA REVISTA ESTÁ PUBLICADA CON EL APOYO INSTITUCIONAL DE REDIRIS-CONSEJO SUPERIOR DE INVESTIGACIONES CIENTIFICAS Y ES DE ACCESO LIBRE. CREATIVE COMMONS LICENSE 4.O BY 